\title{
Video: Totally Laparoscopic Left Lateral Segmentectomy for Hepatic Malignancies: A Modified Technique
}

\author{
Ajay V. Maker • Wisam Jamal • Brice Gayet \\ Published online: 1 June 2011 \\ (C) 2011 The Society for Surgery of the Alimentary Tract
}

\begin{abstract}
We present our series of over 30 totally laparoscopic left hepatic lobectomies (hepatic segments II/III) performed only for malignancy. The short- and long-term results support this technique as safe and efficient. This video will illustrate the pertinent issues regarding trocar placement, intrahepatic anatomy, and the technical maneuvers necessary to perform the modified approach using totally laparoscopic techniques.
\end{abstract}

Keywords Minimally invasive liver surgery $\cdot$ Laparoscopic liver resection · Left lateral segmentectomy · Left hepatic lobectomy · Laparoscopy $\cdot$ Metastasectomy $\cdot$ Segment II $\cdot$ Segment III

\section{Background}

We have been modifying our technique of laparoscopic hepatectomy over the last 16 years. This video demonstrates the relevant technical maneuvers necessary to perform our current modified approach to a totally laparoscopic left hepatic lobectomy (segments II/III).

\section{Methods}

The principal steps of this procedure include laparoscopic ultrasound of the left hepatic vein and portal pedicles to segments II and III, intraparenchymal dissection and division

Dr. Maker was supported in this research by a grant from the Philippe Foundation.

Electronic supplementary material The online version of this article (doi:10.1007/s11605-011-1555-1) contains supplementary material, which is available to authorized users.

A. V. Maker $\cdot$ W. Jamal · B. Gayet

Department of Digestive Diseases, Institut Mutualiste Montsouris, University of Paris V,

Paris, France

\section{A. V. Maker $(\triangle)$}

Division of Surgical Oncology, Department of Surgery,

University of Illinois at Chicago,

Chicago, USA

e-mail: amaker@uic.edu of the segment II and III pedicles, dissection and division of the left hepatic vein, division of the coronary and triangular ligaments, and removal of the specimen. No porta hepatis dissection or pringle maneuver was performed.

\section{Results}

A total of 31 laparoscopic left lateral segmentectomies have been performed successfully at our institution since 1998 for the diagnosis of cancer. Fifteen were performed for colorectal liver metastases, as in the patient in the video, nine for hepatocellular carcinoma, three for diagnosis, one for cholangiocarcinoma, and one each for a renal cell and neuroendocrine metastasis. We have been using the current modified technique since 2006 on the last 20 patients. Our short- and long-term results have been similar to those for our open historical control subjects. The median blood loss was $10 \mathrm{~mL}$, and the median operating time was $115 \mathrm{~min}$. No patients required blood transfusions intraoperatively or postoperatively, and no mortalities occurred. There was one major complication of a biliary leak unable to be managed by interventional drain placement that required a return to the operating room. There were three minor complications of a pleural effusion and two postoperative fevers that resolved with conservative management.

\section{Conclusion}

This modified minimally invasive technique of hepatic resection is a very safe and efficient approach to a left lateral segmentectomy in selected patients with primary or metastatic disease of the liver. 